\title{
Inhibitory effect of PDGF-BB and serum-stimulated responses in vascular smooth muscle cell proliferation by hinokitiol via up-regulation of p21 and p53
}

\author{
Jiun-Yi Li ${ }^{1,2}$, Chun-Ping Liü ${ }^{3}$, Wei-Cheng Shiao ${ }^{4}$, Thanasekaran Jayakumar², Yi-Shin Li², \\ Nen-Chung Chang ${ }^{5}$, Shih-Yi Huang ${ }^{6}$, Cheng-Ying Hsieh²
}

\begin{abstract}
1Department of Cardiovascular Surgery, Mackay Memorial Hospital, and Mackay Medical College, Taipei, Taiwan

${ }^{2}$ Department of Pharmacology and Graduate Institute of Medical Sciences, College of Medicine, Taipei Medical University, Taipei, Taiwan

${ }^{3}$ Department of Cardiology, Yuan's General Hospital, Kaohsiung, Taiwan

${ }^{4}$ Department of Internal Medicine, Yuan's General Hospital, Kaohsiung, Taiwan ${ }^{5}$ Department of Cardiology, School of Medicine, Taipei Medical University, Taipei, Taiwan ${ }^{6}$ School of Nutrition and Health Sciences, Taipei Medical University, Taipei, Taiwan
\end{abstract}

Submitted: 30 April 2015

Accepted: 30 June 2015

Arch Med Sci 2018; 14, 3: 579-587

DOI: https://doi.org/10.5114/aoms.2018.75085

Copyright $\odot 2018$ Termedia \& Banach

\section{Abstract}

Introduction: Vascular smooth muscle cell (VSMC) proliferation plays a major role in the progression of vascular diseases. In the present study, we established the efficacy and the mechanisms of action of hinokitiol, a tropolone derivative found in Chamaecyparis taiwanensis, Cupressaceae, in relation to platelet-derived growth factor-BB (PDGF-BB) and serum-dependent VSMC proliferation.

Material and methods: Primary cultured rat VSMCs were pre-treated with hinokitiol and then stimulated by PDGF-BB $(10 \mathrm{ng} / \mathrm{ml})$ or serum $(10 \% \mathrm{fe}$ tal bovine serum). Cell proliferation and cytotoxicity were determined using the 3-(4,5-dimethylthiazol-2-yl)-2,5-diphenyl tetrazolium bromide assay and lactose dehydrogenase assay, respectively. The degree of DNA synthesis was evaluated by BrdU-incorporation measurements and observed using confocal microscopy. Immunoblotting was utilized to determine the protein level of p-extracellular signal-regulated kinase (ERK) 1/2, p-Akt, p-phosphoinositide 3-kinase (PI3K), p-Janus kinase 2 (JAK2), p-p53, and p21 $1^{\mathrm{Cip} 1}$. The promoter activity of $\mathrm{p} 21$ and $\mathrm{p} 53$ activity were measured by dual luciferase reporter assay.

Results: Treatment with hinokitiol (1-10 $\mu \mathrm{M})$ inhibited PDGF-BB and seruminduced VSMC proliferation and DNA synthesis in a concentration-dependent manner. Cytotoxicity was not observed in hinokitiol-treated VSMCs at the studied concentrations. Pre-incubation of VSMCs with hinokitiol did not alter PDGF-BB-induced phosphorylation of ERK1/2, Akt, PI3K or JAK2. Interestingly, hinokitiol induced promoter activity of p21 and p21 protein expression in VSMCs. Furthermore, hinokitiol augmented $\mathrm{p} 53$ protein phosphorylation and subsequently led to enhanced p53 activity.

Conclusions: These data suggest that the anti-proliferative effects of hinokitiol in VSMCs may be mediated by activation of p21 and p53 signaling pathways, and it may contribute to the prevention of vascular diseases associated with VSMC proliferation.

Key words: vascular smooth muscle cell proliferation, hinokitiol, p21, p53.

\author{
Corresponding author: \\ Dr. Cheng-Ying Hsieh \\ Department \\ of Pharmacology \\ Taipei Medical University \\ $250 \mathrm{Wu}$-Hsing St \\ Taipei 110, Taiwan \\ Phone: +886-2-27361661, \\ ext. 3194 \\ E-mail: hsiehcy@tmu.edu.tw \\ Dr. Shih Y. Huang \\ School of Nutrition \\ and Health Sciences \\ Taipei Medical University \\ $250 \mathrm{Wu}$-Hsing St \\ Taipei 110, Taiwan \\ Phone: +886-2-27361661, \\ ext. 6543 \\ E-mail: sihuang@tmu.edu.tw
}




\section{Introduction}

Increased proliferation of vascular smooth muscle cells (VSMCs) is an important phenomenon in the pathogenesis of atherosclerosis, which is the underlying pathology for many cardiovascular diseases such as myocardial infarction or stroke $[1,2]$. Cell cycle regulation is considered to be an essential mechanism for the inhibition of cell proliferation [3]. The cell cycle is regulated by the synchronized action of cyclin-dependent kinases (CDKs), in association with their specific regulatory cyclin proteins. Therefore, functional activation of CDK-cyclin is required for cell cycle progression $[4,5]$. The kinase activity of these CDK-cyclin complexes is inhibited by two classes of cyclin-dependent kinase inhibitors (CKIs), including the INK4 family (p16INK4a and p15INK4b) and cip family (p21cip1 and p27kip1) [6-8]. p53 is known to be an up-regulator of p21 in cell cycle arrest. Several findings have demonstrated that the product of p53 is responsible for the $\mathrm{G} 1$ checkpoint. In response to genotoxic stress and drugs, the level of p53 protein was increased, and a transient arrest of cell cycle progression in the $\mathrm{G} 1$ phase occurred [9] or apoptosis was triggered [10, 11]. Thus, induction of $\mathrm{p} 53$ has been considered to be an effective strategy for molecular target therapy of atherosclerosis.

Vascular smooth muscle cell proliferation induced by platelet-derived growth factor (PDGF) has been considered to be crucial for the development of vascular diseases. The PDGF interacts with its receptor in VSMCs. PDGF-BB can activate PLC $\gamma$, Akt, and ERK1/2 signaling pathways to induce cell proliferation, cell migration, and angiogenesis of VSMCs [12]. A study has reported that stimulating p53 activity and inducing the expression of p21waf1/cip1 antagonizes the down-regulation of the levels and/or activities of these molecules by PDGF-BB [13]. Therefore, the identification of novel compounds that inhibit PDGF-dependent cell proliferation has the potential to improve existing therapeutic strategies and limit late cardiovascular complications [14].

Hinokitiol (Figure $1 \mathrm{~A}$ ), also known as $\beta$-thujaplicin, is a natural tropolone-related compound found in the heartwood of Cupressaceae plants that has a wide range of biochemical and pharmacological activities [15]. This compound has received more attention from several investigators recently, as it has been used in hair tonics, toothpastes, cosmetics, and food as an antimicrobial agent [16], and several studies have confirmed that it has marked anti-bacterial [17], anti-tumor [18] and neuroprotective activities [19], as well as antioxidant capacities [20]. Recent studies have reported that hinokitiol induces DNA damage and autophagy followed by cell cycle arrest in cancer cells [21,22]. Our previous studies have demonstrated that hinokitiol has potent in vitro and in vivo antithrombotic [23], in vivo neuroprotective [24] and in vitro and in vivo antitumor effects [25]. However, little is known about the effects of hinokitiol on cardiovascular diseases, and the findings of the as sociated signaling mechanisms remain unclear. Therefore, we investigated whether hinokitiol attenuates VSMC proliferation induced by PDGF-BB or serum in vitro.

\section{Material and methods}

\section{Materials}

Hinokitiol (product number: 469521; purity: $\geq$ 98.5\%) and 3-(4,5-dimethylthiazol-2-yl)-2,5-diphenyl tetrazolium bromide (MTT) were purchased from Sigma Chemical Company (St. Louis, MO, USA). Recombinant PDGF-BB was purchased from PeproTech (Rocky Hill, NJ, USA). Anti-mouse and anti-rabbit immunoglobulin G-conjugated horseradish peroxidase (HRP) was purchased from GE Healthcare (Sunnyvale, CA, USA) and/or Jackson-Immuno Research (West Grove, PA, USA). Anti-phospho-ERK1/2 $\left(\mathrm{Thr}^{202} / \mathrm{Tyr}^{204}\right)$, anti-phospho-AKT (Ser ${ }^{473}$ ), antiphospho-PI3K, and antiphospho-JAK2 monoclonal antibodies (mAbs) were purchased from Cell Signaling (Beverly, MA, USA). Anti-p53 was obtained from GeneTex Inc (Irvine, CA). The WWP-luc (p21cip/Waf1 promoter) construct (Addgene plasmid 16451) and the PG13-luc plasmid with p53 binding sites (Addgene plasmid 16642) were kindly provided by Dr. Ming Jen Hsu. The Dual-Glo luciferase assay system was purchased from Promega (Madison, WI). The Hybond-P polyvinylidene difluoride (PVDF) membrane and enhanced chemiluminescence $(E C L)$ Western blotting detection reagent and analysis system were obtained from GE Healthcare (Sunnyvale, CA, USA). All other chemicals used in this study were of reagent grade.

\section{Animal care and cultivation of rat primary VSMCs}

All animal experiments and care were performed according to the National Research Council Guide for the Care and Use of Laboratory Animals, and were approved by the Institutional Animal Care and Use Committee (IACUC) of Taipei Medical University. As previously described [26], VSMCs were enzymatically isolated from the thoracic aortas of male Wistar rats (250-300 g). VSMCs were grown in Dulbecco's modified Eagle's medium (DMEM) supplemented with $20 \mathrm{mM} / \mathrm{I}$ HEPES, 10\% fetal bovine serum (FBS), $1 \%$ penicillin/streptomycin, and $2 \mathrm{mM} / \mathrm{I}$ glutamine at $37^{\circ} \mathrm{C}$ in a humidified atmosphere of $5 \% \mathrm{CO}_{2}$. VSMCs at passage 4-8 were used in all experiments. 
A<smiles>CC(C)c1cccc(=O)c(O)c1</smiles>

B

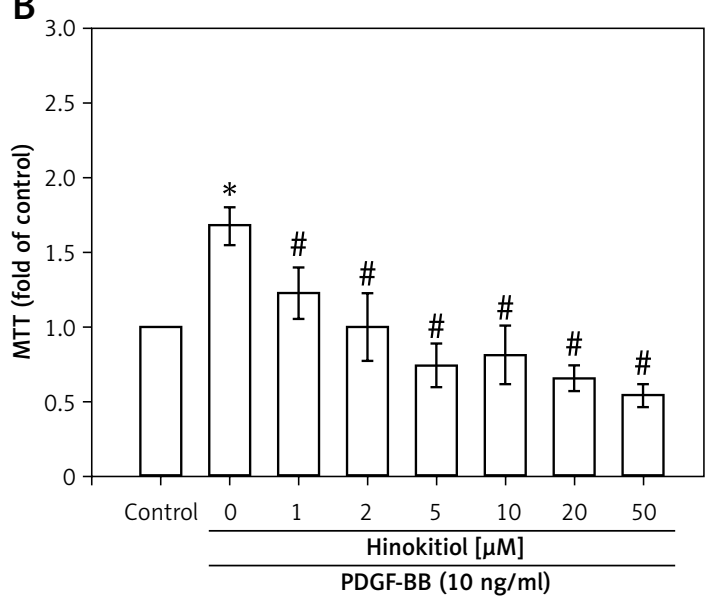

D

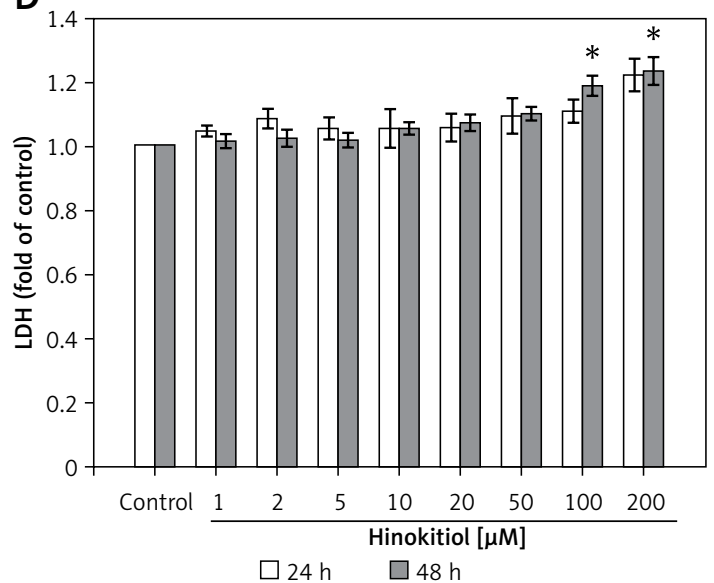

$\square 24 \mathrm{~h} \quad \square 48 \mathrm{~h}$
C

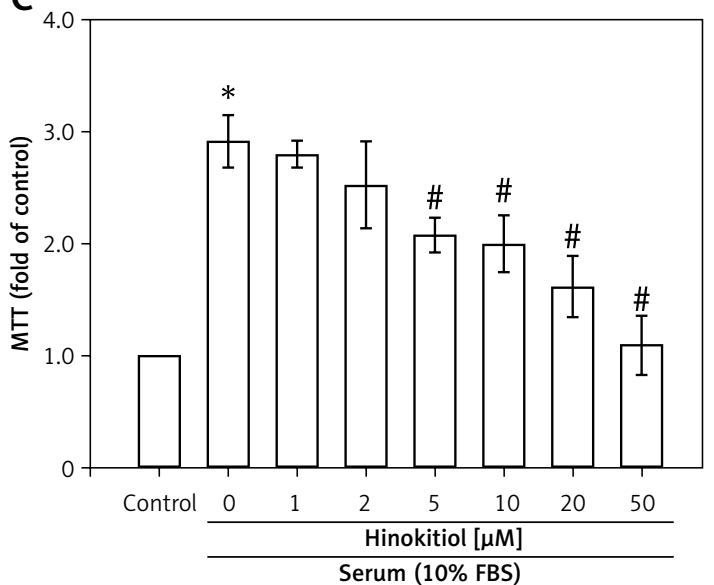

Figure 1. Effects of hinokitiol on platelet-derived growth factor (PDGF)-BB and serum-induced proliferation of vascular smooth muscle cells (VSMCs). A - Chemical structure of hinokitiol. B, C - Serum-starved VSMCs were pretreated in the presence or absence of hinokitiol (1-50 $\mu \mathrm{M}$ ), and then stimulated with (B) $10 \mathrm{ng} / \mathrm{ml} \mathrm{PDGF-BB}$ or (C) $10 \%$ fetal bovine serum (FBS) for $48 \mathrm{~h}$. Cell proliferation was evaluated by MTT assay. D - VSMCs were co-treated with hinokitiol $(1-200 \mu \mathrm{M})$ for 24 or $48 \mathrm{~h}$, and cytotoxicity was determined using the LDH assay. Data are presented as mean $\pm \operatorname{SEM}(n=3) .{ }^{*} p<0.05$ compared to control group, ${ }^{*} p<0.05$ compared to PDGF-BB or FBS stimulated cells

\section{MTT assay}

VSMCs $\left(2 \times 10^{4}\right.$ cells/well $)$ were seeded on 24 well plates and cultured in DMEM containing $10 \%$ FBS for $24 \mathrm{~h}$. The cell was then treated according to the experimental designs. The cell number was measured using a colorimetric assay based on the ability of mitochondria in viable cells to reduce the MTT as previously described [27]. The cell number index was calculated as the absorbance of treated cells/control cells.

\section{Lactose dehydrogenase (LDH) assay}

Cytotoxicity was assessed by LDH assay in the supernatant medium using a non-radioactive cytotoxicity kit according to the protocol of the manufacturer (BioVision Inc, Milpitas, CA, USA). VSMCs were seeded on a round-bottom 96-well culture plate in triplicate sets of wells. After incubation, cells were treated with various concentrations of hinokitiol $(1-200 \mu \mathrm{M})$ for 24 and 48 h. VSMCs were lysed followed by two freeze/thaw cycles. The plate was centrifuged at $250 \mathrm{~g}$ for $4 \mathrm{~min}$, and $50 \mu \mathrm{l}$ of supernatant was transferred from each well to a fresh 96-well flat-bottom plate. Then $50 \mu$ of the reconstituted substrate mix was added to each well of the plate. The plate was covered with foil to protect it from light, and then the plate was incubated at room temperature for $30 \mathrm{~min}$. The reaction was stopped by the addition of $50 \mu$ of stop solution, and LDH was determined by measuring the absorbance at $490 \mathrm{~nm}$. The fold activity of LDH was calculated from the following equation: [(experimental LDH release)/(vehicle LDH release)].

\section{DNA synthesis assay}

VSMCs $\left(2 \times 10^{5}\right.$ cells/dish) were seeded in a 96well microplate for $24 \mathrm{~h}$ and then serum-starved for $24 \mathrm{~h}$. Following preincubation with hinokitiol for $20 \mathrm{~min}$, the cells were treated with PDGF-BB $(10 \mathrm{ng} / \mathrm{ml})$ and $10 \%$ FBS serum for $48 \mathrm{~h}$. DNA syn- 
thesis was assessed using BrdU incorporation assay kits (Roche Diagnostics, Rotkreuz, Switzerland) according to the manufacturer's instructions. DNA synthesis in VSMCs was assessed by the incorporation of BrdU. In addition, confocal microscopy was also used to observe the expression of BrdU positive cells. VSMCs $\left(1 \times 10^{5}\right.$ cells/cover slip) were placed on cover slips and allowed to adhere in a cell culture incubator overnight. VSMCs were treated as experimental design, and fixed with BrdU fixed solution (Roche Diagnostics, Rotkreuz, Switzerland). After incubation with $3 \%$ bovine serum albumin (BSA) in phosphate-buffered saline (PBS) for $60 \mathrm{~min}$, the preparation was incubated overnight with a primary anti-BrdU Ab (1:80). Cells were then washed three times with PBS and exposed to goat anti-mouse IgG secondary antibody, Alexa Fluor 488 conjugate $(1: 1000)$ (Thermo Fisher Scientific, Waltham, MA, USA) for $2 \mathrm{~h}$. The samples were counter-stained with DAPI and mounted with mounting buffer (Vector Laboratories, Burlingame, CA, USA) under a glass cover slip on a Leica TCS SP5 Confocal Spectral Microscope Imaging System using an argon/krypton laser (Mannheim, Germany).

\section{Immunoblotting}

Immunoblotting was performed as previously described [28]. Serum-starved VSMCS $\left(2 \times 10^{5}\right.$ cells/dish) were treated with hinokitiol $(1-100 \mu \mathrm{M})$ or DMSO for $20 \mathrm{~min}$, followed by the addition of PDGF-BB $(10 \mathrm{ng} / \mathrm{ml})$ for the indicated times. After treatment, proteins were extracted with lysis buffer. The lysates were centrifuged, the supernatant protein $(50 \mu \mathrm{g})$ was collected and subjected to sodium dodecylsulfate polyacrylamide gel electrophoresis (SDS-PAGE), and the separated proteins were electrophoretically transferred onto $0.45-\mu \mathrm{m}$ polyvinylidene difluoride (PVDF) membranes. The blots were blocked with TBST (10 mM Tris-base, $100 \mathrm{mM} \mathrm{NaCl}$, and $0.01 \%$ Tween 20) containing $5 \%$ bovine serum albumin (BSA) for $1 \mathrm{~h}$ and were then probed with various primary antibodies. The membranes were incubated with HRP-linked anti-mouse IgG or anti-rabbit IgG (diluted $1: 3000$ in TBST) for $1 \mathrm{~h}$. Immunoreactive bands were detected by an enhanced chemiluminescence $(E C L)$ system. The bar graph depicts the ratios of quantitative results obtained by scanning reactive bands and quantifying the optical density using video densitometry (Bio-profil; Biolight Windows Application V2000.01; Vilber Lourmat, France).

\section{Dual luciferase reporter assay}

Cells were transfected with PG13-luc or p21cip/ Waf1-luc using Turbofect reagent (Upstate Biotechnology, Lake Placid, NY). Cells with and without treatments were then harvested, and the luciferase activity was determined using a Dual-Glo luciferase assay system kit (Promega, Madison, WI, USA) according to the manufacturer's instructions. Normalization was performed with Renilla luciferase activity as the basis.

\section{Statistical analysis}

The experimental results are expressed as mean \pm SEM. One-way analysis of variance (ANOVA) was used for multiple comparisons (Sigma Stat v3.5 software). If there was significant variation between treated groups, the Student-Newman-Keuls test was applied. A $p$-value $<0.05$ was considered to be statistically significant.

\section{Results}

Hinokitiol inhibits PDGF-BB and serum induced proliferation of VSMCS

PDGF-BB $(10 \mathrm{ng} / \mathrm{ml})$ or serum $(10 \%$ FBS $)$ induced VSMC proliferation in a concentration-dependent manner (Figures $1 \mathrm{~B}, \mathrm{C}$ ). In addition, it was found that VSMC pretreated with hinokitiol inhibits cell proliferation after both PDGF-BB and serum stimulation in a concentration-dependent manner. These results suggest that the inhibitory effects of hinokitiol on VSMC proliferation are not specific to PDGF-BB. As anti-proliferative activity is regularly conveyed by cytotoxicity, this study also measured the cytotoxic effects of hinokitiol on VSMCs, and found no evidence of cytotoxicity up to $50 \mu \mathrm{M}$ in both 24 and 48 h (Figure 1 D) incubation. Thus, these results indicated that hinokitiol appeared to inhibit VSMC proliferation without cytotoxicity.

\section{Hinokitiol inhibits PDGF-BB and serum induced DNA synthesis in VSMCs}

The BrdU incorporation assay was used to further investigate the effects of hinokitiol on PDGF-BB and serum stimulated DNA synthesis. The results revealed that hinokitiol significantly inhibited the BrdU incorporation induced by PDGF-BB and serum stimulation in a dose-dependent manner (Figures $2 \mathrm{~A}, \mathrm{~B})$. Moreover, Figure $2 \mathrm{C}$ also shows that the treatment of hinokitiol $(10 \mu \mathrm{M})$ significantly reduces the BrdU positive VSMCs induced by PDGF-BB through the immunofluorescence staining assay. These results indicate that the inhibitory effect of hinokitiol targets DNA synthesis rather than cytotoxicity to cause the loss of cellular DNA.

\section{Role of hinokitiol in signaling pathways induced by PDGF-BB}

We next examined the signaling pathway(s) involved in the inhibitory effect of hinokitiol on VSMC proliferation in response to PDGF-BB stimulation. The phosphorylation levels of ERK1/2, Akt, PI3K and JAK2 were increased by PDGF-BB treat- 


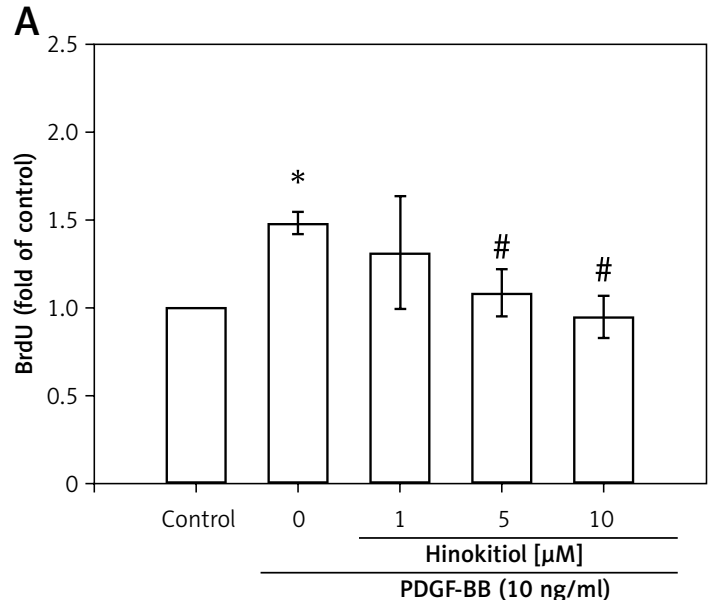

C
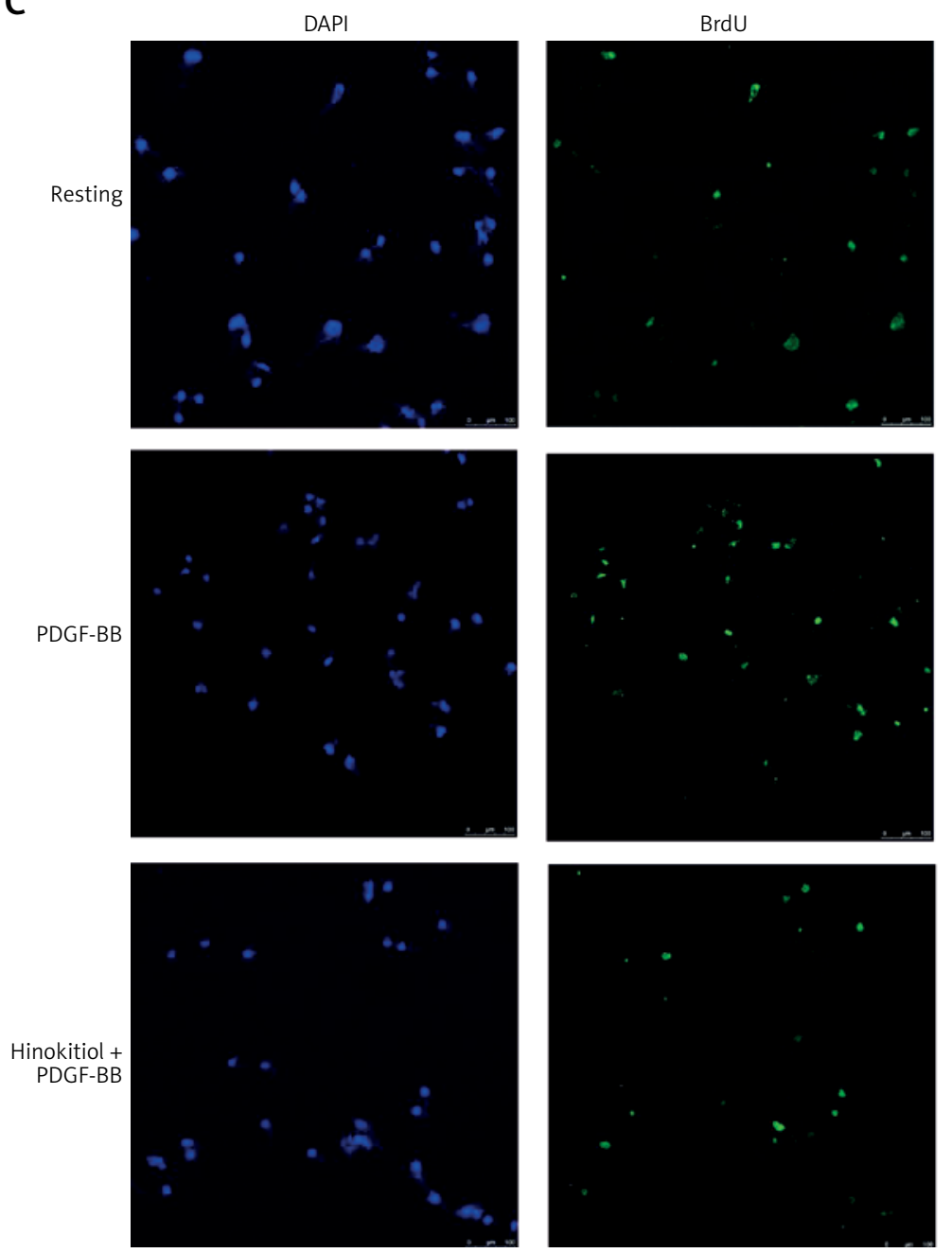
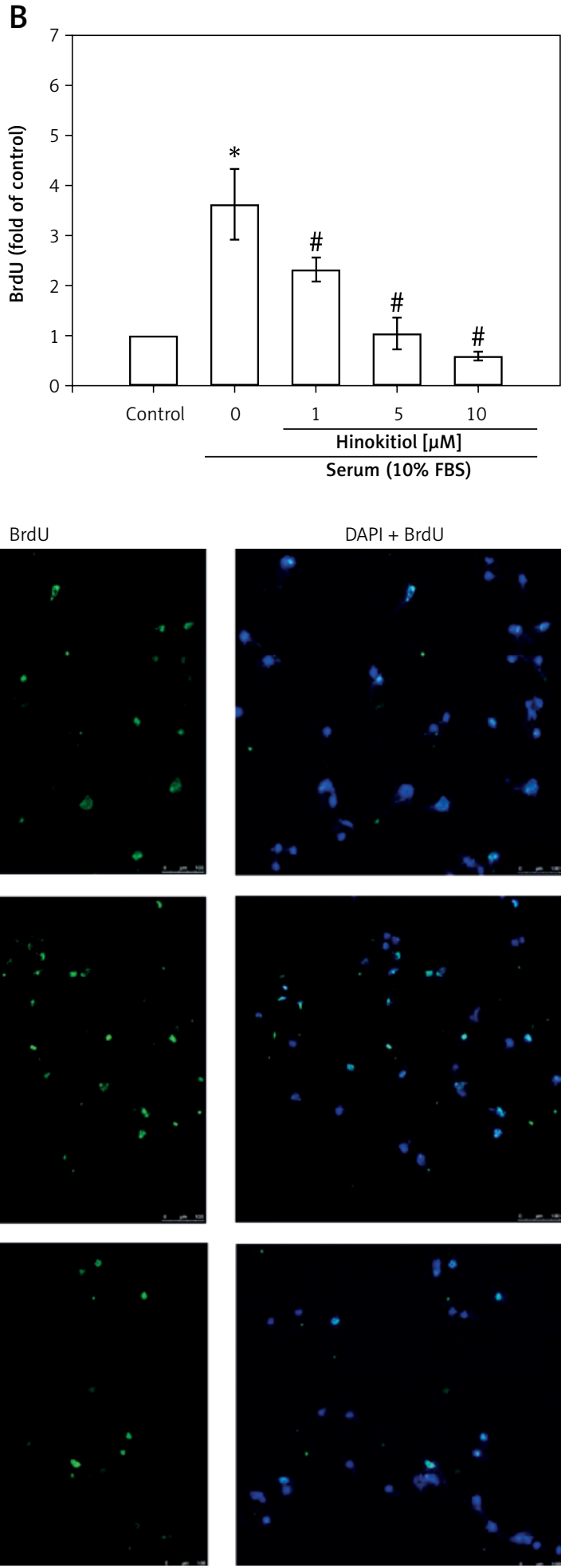

Figure 2. Hinokitiol inhibits PDGF-BB and serum induced DNA synthesis in VSMCs. Serum-starved VSMCs were pretreated in the presence or absence of hinokitiol (1-10 $\mu \mathrm{M}$ ), and then stimulated with (A) $10 \mathrm{ng} / \mathrm{ml}$ PDGF-BB or (B) $10 \%$ fetal bovine serum (FBS) for $48 \mathrm{~h}$. DNA synthesis was assessed using BrdU incorporation assay kits. Data are presented as mean \pm SEM $(n=3) .{ }^{*} p<0.05$ compared to control group, ${ }^{*} p<0.05$ compared to PDGF-BB or FBS stimulated cells. C - VSMCs $\left(1 \times 10^{5}\right.$ cells/cover slip) were placed on cover slips, allowed to adhere overnight and then serum starved for $24 \mathrm{~h}$. The VSMCs were pre-treated in the presence or absence of hinokitiol $(10 \mu \mathrm{M})$, and then stimulated with $10 \mathrm{ng} / \mathrm{ml}$ PDGF-BB for $48 \mathrm{~h}$. BrdU positive VSMCs were directly observed by confocal microscopy. Representative immunofluorescence staining for BrdU was revealed in green and nuclear staining DAPI was revealed in blue. The white bar indicates $100 \mu \mathrm{m}$ 
ment, whereas these modifications were not significantly reversed by hinokitiol treatment. The total protein levels of ERK and Akt and $\alpha$-tubulin did not change during the course of stimulation with PDGF-BB in the presence or absence of hinokitiol (Figures $3 \mathrm{~A}-\mathrm{D}$ ). These data suggest that ERK1/2, Akt, PI3K and JAK2 signaling pathways are not involved in the anti-proliferative effects of hinokitiol in VSMCs in response to PDGF-BB.

\section{Hinokitiol induces p21 expression in VSMCs}

p21 was found to be up-regulated concentration dependently in VSMCs in response to hinokitiol treatment (Figure $4 \mathrm{~A}$ ). A representative luciferase assay for p21 is shown in Figure 4 B. Hinokitiol at a dose of $5 \mu \mathrm{M}$ significantly $(p<0.05)$ increased p21 protein expression in VSMCs.

Hinokitiol induces p53 phosphorylation and its binding effects in VSMCs

As p53 is essential for proliferation processes in VSMCs, the effect of hinokitiol on p53 phos- phorylation was assessed. Figure $4 \mathrm{C}$ shows that treatment of VSMC with hinokitiol $(5 \mu \mathrm{M})$ for 10 and 20 min caused enhanced phosphorylation of p53, whereas it is down-regulated at 30 and $60 \mathrm{~min}$ treatment periods. The p53 luciferase reporter gene assay demonstrated that p53 promoter activity was induced after treatment with hinokitiol (Figure $4 \mathrm{D}$ ).

\section{Discussion}

Abnormal proliferation of VSMCs in arterial walls acts as a critical contributing factor in the pathogenesis of atherosclerosis and restenosis after angioplasty, and also in the development of hypertension [29]. We demonstrated that hinokitiol potently inhibited PDGF-BB and serum-stimulated proliferation by increasing inhibition of newly synthesized DNA without affecting apoptosis in VSMCs. Hinokitiol up-regulated the expression level of $\mathrm{p} 21$, the upstream regulatory protein in VSMCs.

p21 $1^{\text {Cip1 }}$, an important member of the CIP/KIP family of CDKIs, has been described to specifically normalize the cell cycle transition [30], and it in-

A

B
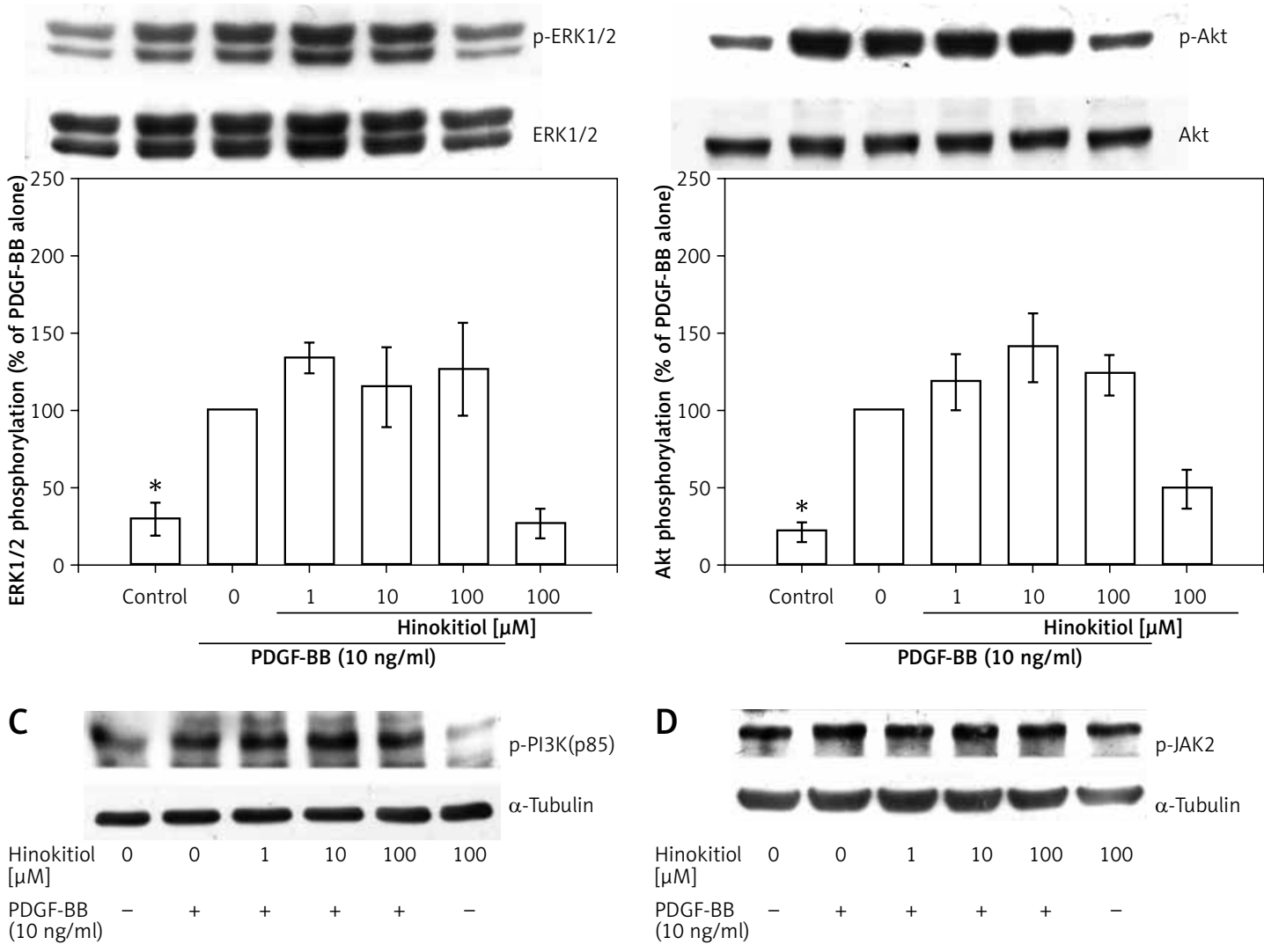

Figure 3. Effect of hinokitiol on PDGF-BB signaling pathway in VSMCs. A - Confluent cells were pre-treated in the presence or absence of hinokitiol $(1-100 \mu \mathrm{M})$ in a serum-free medium, and then stimulated with $10 \mathrm{ng} / \mathrm{ml}$ PDGF-BB for 10 min. The cells were lysed, and the levels of (A) p-ERK1/2, (B) p-Akt, (C) p-PI3K and (D) p-JAK2 were analyzed by SDS-PAGE and immunoblotting. The respective total proteins and $\alpha$-tubulin were used for the normalization of phosphorylated ones. Data are expressed as mean \pm SEM $(n=3) .{ }^{*} p<0.05$ compared to the PDGF-BB stimulated cells 
A

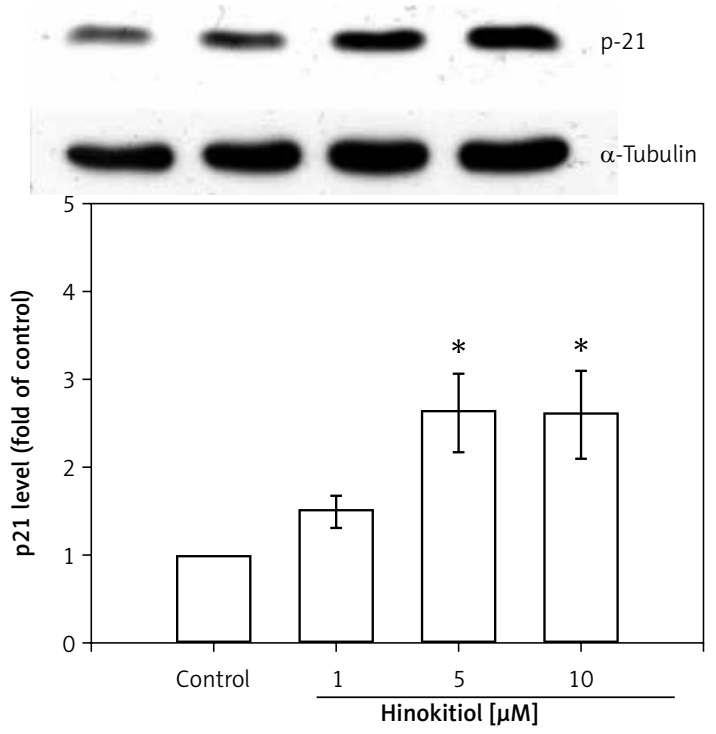

C
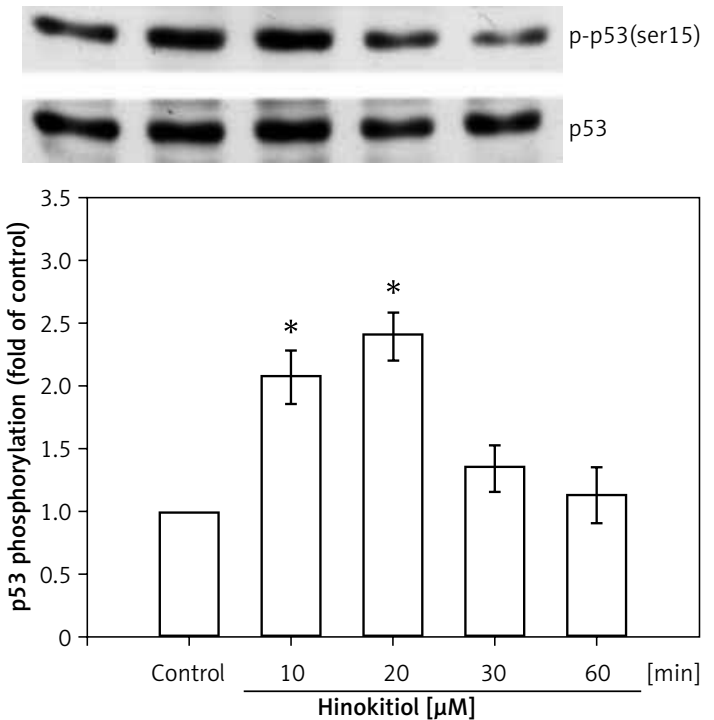

B

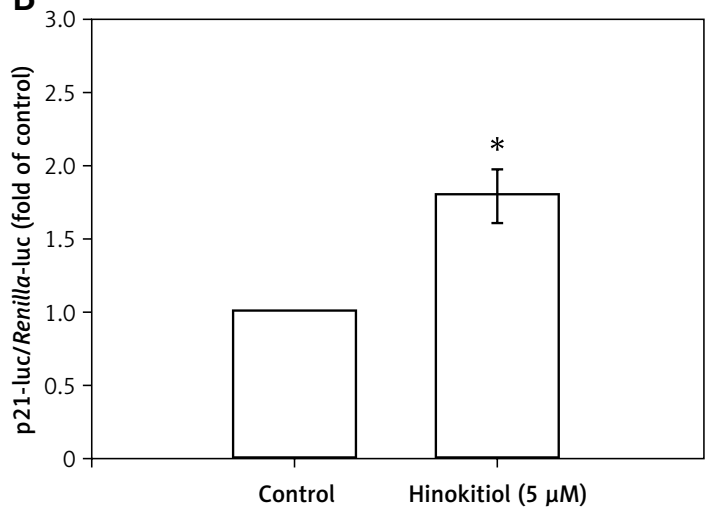

D

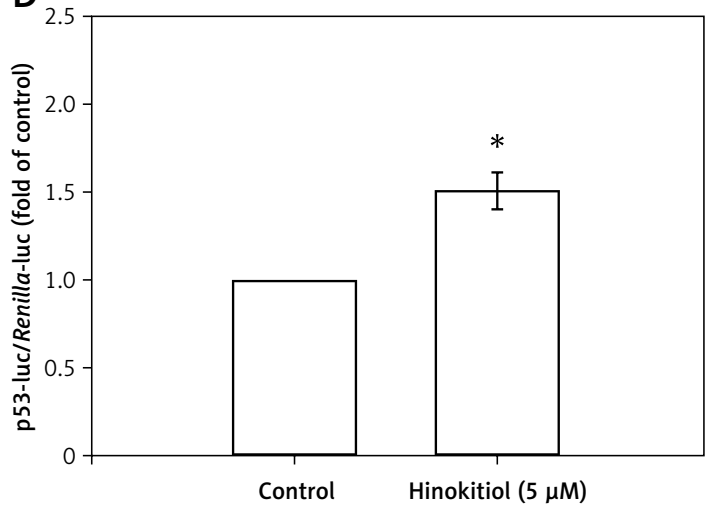

Figure 4. Effect of hinokitiol on p21 expression and p53 activation in VSMCs. A, C - VSMCs were pre-treated in the presence or absence of hinokitiol for $24 \mathrm{~h}(\mathbf{A})$ or the indicated time period (C). The cells were then lysed, and the level of p21 (A) and p-p53 (C) was analyzed by immunoblotting. B, D - VSMCs were transiently transfected with p21cip/Waf1-luc (B) or PG-13-luc (D) and Renilla-luc for 48 h. Transfected VSMCs were pre-treated in the presence or absence of hinokitiol for $24 \mathrm{~h}$. The luciferase activity was then determined. Data are expressed as mean \pm S.E.M; $(n=3),{ }^{*} p<0.05$ compared to the control group

hibits G1 cyclin/Cdks activity by blocking cell cycle progression, such as cyclin E- and cyclin A-CDK2 complexes [31, 32]. In addition, p2 $1^{\text {cip } 1}$ participates in modulation of cell differentiation and apoptosis [6]. In the western blot analysis, we observed that the expression of p21 was significantly and dose dependently increased by hinokitiol treatment. Elevated p21 led to a marked increase in p21 promoter gene expression. Consequently, it can be proposed that p21 is another potential effector to control the G1/S transition. Transcriptional regulation of p21 is known to occur via both p53 dependent and independent mechanisms [33]. In the conventional p53 dependent pathway,
DNA damage results in p53 activation. p53 then interacts with response elements present on the promoter region of p21 to increase expression of p21. Normally, p21 acts as a major effector of p53 to control the G1 cell cycle checkpoint [8]. In the present study, the results also showed that the upregulation of the $\mathrm{p} 53$ protein phosphorylation by hinokitiol led to increased p53 luciferase reporter gene overexpression. These results may suggest that hinokitiol potentially blocks VSMC proliferation via activation of $\mathrm{p} 53$ and $\mathrm{p} 21$.

The current study further investigated the effects of hinokitiol on the PDGF-signaling pathway in PDGF-BB-stimulated VSMCS. PDGF is a potent 


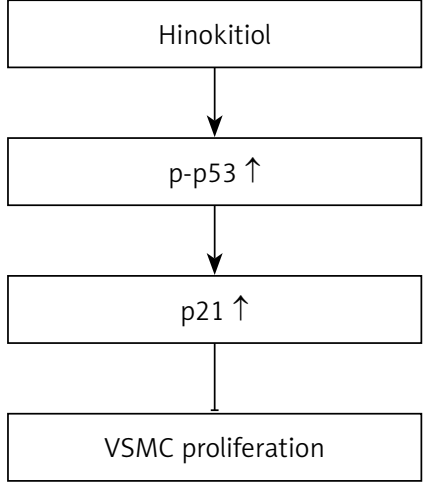

Figure 5. Hypothetical scheme of the inhibitory mechanism of hinokitiol in VSMC proliferation

growth factor produced by VSMCs, endothelial cells, and platelets in injured vascular wall [12, 34]. After PDGF-BB is bound to its receptor in smooth muscle cells, three major signal transduction pathways - p-PLC $\gamma 1, p$-AKt, and p-p-ERK1/2 - can be activated [35]. In this study, we found that hinokitiol had no effect on the expression of p-ERK1/2, p-Akt, and p-JAK2. Therefore, these results suggest that hinokitiol may inhibit the PDGF-BB or serum-stimulated proliferation of VSMCs, through enhancing the p21 and p53 signaling pathway.

$\mathrm{PI} 3 \mathrm{~K} / \mathrm{Akt}$ is known as one of the principal signaling molecules for cell proliferation and survival mediated by extracellular stimuli [36]. Substances for inhibition of the PI3K/Akt pathway such as AS605240, TG100-115, PIK-75 and TGX-221 have been widely studied in treatment of hypertension and angina, and show an array of biological effects in the cardiovascular system [37]. The PI3K/Akt inhibitors have also been used widely as pharmaceutical tools and associated signaling pathways in essential biologic processes $[38,39]$. The insights into the role of PI3K/Akt in human diseases provide a wide spectrum of therapeutic strategies. It has been reported that PI3K/Akt is highly expressed in human as well as murine atherosclerotic lesions, and the pharmacological PI3K inhibitor AS605240, 5-quinoxilin-6-methylene-1,3-thiazolidine-2,4-dione, significantly reduced early atherosclerotic lesions in apolipoprotein E (Apo-E)-null mice [40]. A similar effect was also observed in an atherosclerotic lesion of LDL receptor-deficient mice in response to AS605240, suggesting that this protein is a promising target for the treatment of atherosclerosis [39]. In addition, topotecan, a water soluble camptothecin analog, inhibits vascular endothelial growth factor (VEGF) and basic fibroblast growth factor (bEGF)induced vascular endothelial cell migration via downregulation of the PI3K/Akt signaling pathway [41]. In contrast, the results of the current study show that hinokitiol treatment did not alter the expression of PDGF-BB-induced PI3K. It evidently confirms that hinokitiol inhibits PDGF-BB-stimulated VSMC proliferation, through enhancing the p21 and p53 signaling pathway (Figure 5).

In conclusion, these results suggest that hinokitiol may inhibit vascular smooth muscle cell proliferation via the $\mathrm{p} 21 / \mathrm{p} 53$ signaling pathway, followed by inhibiting DNA synthesis. Proliferation of vascular smooth muscle cells has been identified to play a critical role in the pathogenesis of atherosclerosis. Hinokitiol may be a promising candidate for the treatment of atherosclerosis and related cardiovascular diseases.

\section{Acknowledgments}

Dr. Jiun-Yi Li and Dr. Chun-Ping Liu contributed equally to this work.

This work was supported by grants from the National Science Council of Taiwan (NSC97-2320B-038-016-MY3 and NSC100-2320-B-038-021-MY3), and Yuan's General Hospital-Taipei Medical University (103-YGH-TMU-04; 103-YGH-TMU-01-2).

\section{Conflict of interest}

The authors declare no conflict of interest.

\section{References}

1. Doran AC, Meller N, McNamara CA. Role of smooth muscle cells in the initiation and early progression of atherosclerosis. Arterioscler Thromb Vasc Biol 2008; 28: 812-9.

2. Owens GK, Kumar MS, Wamhoff BR. Molecular regulation of vascular smooth muscle cell differentiation in development and disease. Physiol Rev 2004; 84: 767-801.

3. Dzau VJ, Braun-Dullaeus RC, Sedding DG. Vascular proliferation and atherosclerosis: new perspectives and therapeutic strategies. Nat Med 2002; 8: 1249-56.

4. Braun-Dullaeus RC, Mann MJ, Sedding DG, et al. Cell cycle-dependent regulation of smooth muscle cell activation. Arterioscler Thromb Vasc Biol 2004; 24: 845-50.

5. Xiong $\mathrm{Y}$, Hannon GJ, Zhang $\mathrm{H}$, et al. p21 is a universal inhibitor of cyclin kinases. Nature 1993; 366: 701-4.

6. Coqueret 0 . New roles for p21 and p27 cell-cycle inhibitors: a function for each cell compartment? Trends Cell Biol 2003; 13: 65-70.

7. Ortega S, Malumbres M, Barbacid M, et al. Cyclin D-dependent kinases, INK4 inhibitors and cancer. Biochim Biophys Acta 2002; 1602: 73-87.

8. Sherr CJ, Roberts JM. CDK inhibitors: positive and negative regulators of $\mathrm{G} 1$-phase progression. Genes Dev 1999; 13: 1501-12.

9. Macleod KF, Sherry N, Hannon G, et al. p53-dependent and independent expression of p21 during cell growth, differentiation, and DNA damage. Genes Dev 1995; 9: 935-44.

10. Chan KC, Ho HH, Peng $\mathrm{CH}$, et al. Polyphenol-rich extract from mulberry leaf inhibits vascular smooth muscle cell proliferation involving upregulation of p53 and inhibition of cyclin-dependent kinase. J Agric Food Chem 2010; 58: 2536-42.

11. Pellegata NS, Antoniono RJ, Redpath JL, et al. DNA dam age and p53-mediated cell cycle arrest: a reevaluation. Proc Natl Acad Sci USA 1996; 93: 15209-14. 
12. Chaudhary LR, Hruska KA. The cell survival signal Akt is differentially activated by PDGF-BB, EGF, and FGF-2 in osteoblastic cells. J Cell Biochem 2001; 81: 304-11.

13. Bhanoori M, Yellaturu CR, Ghosh SK, et al. Thiol alkylation inhibits the mitogenic effects of platelet-derived growth factor and renders it proapoptotic via activation of STATs and $\mathrm{p} 53$ and induction of expression of caspase 1 and p21waf1/cip1. Oncogene 2003; 22: 117-30.

14. Levitzki A. PDGF receptor kinase inhibitors for the treatment of restenosis. Cardiovasc Res 2005; 65: 581-6.

15. Zhao J, Fujita K, Sakai K. Reactive oxygen species, nitric oxide, and their interactions play different roles in Cupressus lusitanica cell death and phytoalexin biosynthesis. New Phytol 2007; 175: 215-29.

16. Saeki Y, Ito $Y$, Shibata $M$, et al. Antimicrobial action of natural substances on oral bacteria. Bull Tokyo Dent Coll 1989; 30: 129-35

17. Arima Y, Nakai Y, Hayakawa R, et al. Antibacterial effect of beta-thujaplicin on staphylococci isolated from atopic dermatitis: relationship between changes in the number of viable bacterial cells and clinical improvement in an eczematous lesion of atopic dermatitis. J Antimicrob Chemother 2003; 51: 113-22.

18. Morita Y, Matsumura E, Okabe T, et al. Biological activity of beta-dolabrin, gamma-thujaplicin, and 4-acetyltropolone, hinokitiol-related compounds. Biol Pharm Bull 2004; 27: 1666-9.

19. Koufaki M, Theodorou E, Alexi X, et al. Synthesis of tropolone derivatives and evaluation of their in vitro neuroprotective activity. Eur J Med Chem 2010; 45: 1107-12.

20. Liu S, Yamauchi H. p27-Associated G1 arrest induced by hinokitiol in human malignant melanoma cells is mediated via down-regulation of pRb, Skp2 ubiquitin ligase, and impairment of Cdk2 function. Cancer Lett 2009; 286: 240-9.

21. Li LH, Wu P, Lee JY, et al. Hinokitiol induces DNA damage and autophagy followed by cell cycle arrest and senescence in gefitinib-resistant lung adenocarcinoma cells. PLoS One 2014; 9: e104203.

22. Wang WK, Lin ST, Chang WW, et al. Hinokitiol induces autophagy in murine breast and colorectal cancer cells. Environ Toxicol 2016; 31: 77-84.

23. Lin KH, Kuo JR, Lu WJ, et al. Hinokitiol inhibits platelet activation ex vivo and thrombus formation in vivo. Biochem Pharmacol 2013; 85: 1478-85.

24. Jayakumar T, Hsu WH, Yen TL, et al. Hinokitiol, a natural tropolone derivative, offers neuroprotection from thromboembolic stroke in vivo. Evid Based Complement Alternat Med 2013; 2013: 840487.

25. Huang $\mathrm{CH}$, Chang CC, Thomas PA, et al. Hinokitiol, a tropolone derivative, inhibits mouse melanoma (B16-F10) cell migration and in vivo tumor formation. Eur J Pharmacol 2015; 746: 148-57.

26. Chang $\mathrm{Y}$, Uen $\mathrm{YH}$, Chen $\mathrm{CC}$, et al. Platonin inhibited PDGF-BB-induced proliferation of rat vascular smooth muscle cells via JNK1/2-dependent signaling. Acta Pharmacol Sin 2011; 32: 1337-44.

27. Hsieh CY, Liu CL, Hsu MJ, et al. Inhibition of vascular smooth muscle cell proliferation by the vitamin E derivative pentamethylhydroxychromane in an in vitro and in vivo study: pivotal role of hydroxyl radical-mediated PLCgamma1 and JAK2 phosphorylation. Free Radic Bio Med 2010; 49: 881-93.

28. Wang JJ, Huan SK, Hsieh KH, et al. Inhibitory effect of midazolam on MMP-9, MMP-1 and MMP-13 expression in PMA-stimulated human chondrocytes via recovery of NF-kappaB signaling. Arch Med Sci 2013; 9: 332-9.
29. Lim Y, Kwon JS, Kim DW, et al. Obovatol from Magnolia obovate inhibits vascular smooth muscle cell proliferation and intimal hyperplasia by inducing p21Cip1. Atherosclerosis 2010; 210: 372-80.

30. Philipp-Staheli J, Kim KH, Liggitt D, et al. Distinct roles for p53, p27Kip1, and p21Cip1 during tumor development. Oncogene 2004; 23: 905-13.

31. Harper JW, Adami GR, Wei N, et al. The p21 Cdk-interacting protein Cip1 is a potent inhibitor of $\mathrm{G} 1$ cyclin-dependent kinases. Cell 1993; 75: 805-16.

32. Stewart ZA, Leach SD, Pietenpol JA. p21(Waf1/Cip1) inhibition of cyclin E/Cdk2 activity prevents endoreduplication after mitotic spindle disruption. Mol Cell Biol 1999; 19: 205-15

33. Gartel AL, Tyner AL. Transcriptional regulation of the $p 21$ (WAF1/CIP1) gene. Exp Cell Res 1999; 246: 280-9.

34. Majesky MW, Reidy MA, Bowen-Pope DF, et al. PDGF ligand and receptor gene expression during repair of arterial injury. J Cell Biol 1990; 111: 2149-58.

35. Kim JH, Jin YR, Park BS, et al. Luteolin prevents PDGF-BBinduced proliferation of vascular smooth muscle cells by inhibition of PDGF beta-receptor phosphorylation. Biochem Pharmacol 2005; 69: 1715-21.

36. Parcellier A, Tintignac LA, Zhuravleva E, et al. PKB and the mitochondria: AKTing on apoptosis. Cell Signal 2008; 20: 21-30.

37. Eisenreich A, Rauch U. PI3K inhibitors in cardiovascular disease. Cardiovasc Ther 2011; 29: 29-36.

38. Cantley LC. The phosphoinositide 3-kinase pathway. Science 2002; 296: 1655-7.

39. Maira SM, Stauffer F, Schnell C, et al. PI3K inhibitors for cancer treatment: where do we stand? Biochem Soc Trans 2009; 37: 265-72.

40. Fougerat A, Gayral S, Gourdy P, et al. Genetic and pharmacological targeting of phosphoinositide 3-kinasegamma reduces atherosclerosis and favors plaque stability by modulating inflammatory processes. Circulation 2008; 117: 1310-7.

41. Nakashio A, Fujita N, Tsuruo T. Topotecan inhibits VEGFand bFGF-induced vascular endothelial cell migration via down-regulation of the PI3K-Akt signaling pathway. Int J Cancer 2002; 98: 36-41. 